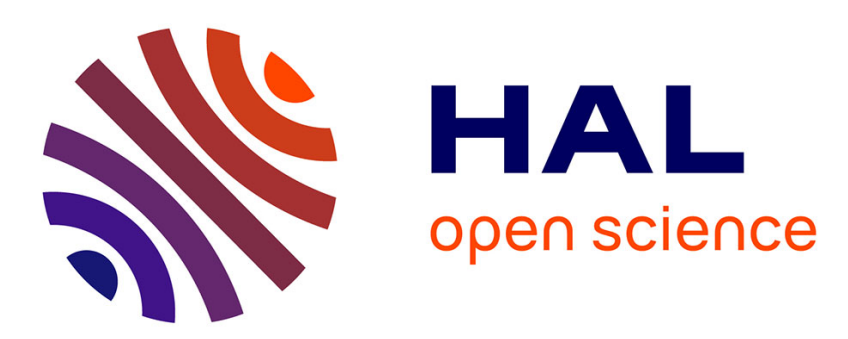

\title{
Formulation of wave propagation in infinite media by normal coordinates with an application to diffraction
}

\author{
Maurice A. Biot, I. Tolstoy
}

\section{To cite this version:}

Maurice A. Biot, I. Tolstoy. Formulation of wave propagation in infinite media by normal coordinates with an application to diffraction. Journal of the Acoustical Society of America, 1957, 29 (3), pp.381391. 10.1121/1.1908899 . hal-01368679

\section{HAL Id: hal-01368679 \\ https://hal.science/hal-01368679}

Submitted on 21 Sep 2016

HAL is a multi-disciplinary open access archive for the deposit and dissemination of scientific research documents, whether they are published or not. The documents may come from teaching and research institutions in France or abroad, or from public or private research centers.
L'archive ouverte pluridisciplinaire HAL, est destinée au dépôt et à la diffusion de documents scientifiques de niveau recherche, publiés ou non, émanant des établissements d'enseignement et de recherche français ou étrangers, des laboratoires publics ou privés. 


\title{
Formulation of Wave Propagation in Infinite Media by Normal Coordinates with an Application to Diffraction*
}

\author{
M. A. Biot, $\nmid$ Shell Development Company, New York, New York
}

AND

\author{
I. Tolstoy, Columbia University, Hudson Laboratories, Dobbs Ferry, New York
}

(Received August 31, 1956)

In the theories of acoustical and electromagnetic vibrations of enclosures, as well as in field theory and electrodynamics, one quite commonly uses normal modes as generalized coordinates in Hilbert space. Here the method is extended to unlimited or partially limited mechanical media, essentially by first solving the problem for an enclosure and going to a limit while expanding all or some of the boundaries to infinity. This leads to a very useful technique, of somewhat more generality than analogous procedures used in field theory. Thus it is applicable to all nondissipative mechanical continua, for any boundary conditions, irrespective of the order or number of differential operators describing the continua and regardless of whether the coordinate systems are separable or not.

A general orthonormality condition valid in all such cases together with the necessary rules for dealing with divergent normalizing coefficients are easily obtained by limiting procedures. An equally general formulation for arbitrary sources is obtained from the principle of virtual work. The method is illustrated by simple examples, for the case of a point source representing the instantaneous injection of a unit volume, i.e., an idealized, infinitely rapid, explosion. It is shown that in the problem of diffraction by a rigid wedge or corner one is led very quickly to an explicit solution in closed form, involving elementary functions only. Some physical implications of this solution are mentioned briefly. The advantages of the normal coordinates method are discussed when thus used in propagation or diffraction problems in unlimited or partially limited mechanical continua. Obvious advantages are its generality, its flexibility in dealing with arbitrary sources, and the fact that it leads directly to the progressive transient solution.

\section{INTRODUCTION}

$T$ HE use of normal modes as generalized coordinates is a well-established practice in mathematical physics and applied mathematics. Elementary applications deal with discrete systems of masses and springs, molecules and electric circuits, and with the electromagnetic or acoustical vibrations of enclosures. It is also used in field theory, in the context of infinite spaces. ${ }^{1,2}$ The range of problems to which it may be applied is very great since it can be adapted to all physical systems that are uniquely defined by two positive definite quadratic forms.

In this paper we extend the technique to the case of unlimited or partially limited mechanical media. In so doing we have of course at our disposal a wealth of known results which only require some generalization and refinement to be applicable to all problems in nondissipative acoustics, elasticity and complex coupled wave problems. The procedure followed is, in principle, to solve first the problem for a finite system and then go to a limit while expanding all or some of the boundaries to infinity. This technique is reminiscent of field theoretical methods, but is more general. It can be applied to a broad class of problems characterized by difficulties of a type not encountered in field theory, such as cases requiring the use of higher order operators and nonstandard boundary conditions (e.g., prestressed, inhomogeneous, anisotropic elastic solids). For most

\footnotetext{
* The present work was carried out under the sponsorship of the Shell Development Company for the Exploration and Production Research Division.

$\dagger$ Consultant.

$1 \mathrm{~W}$. Heitler, The Quantum Theory of Radiation (Clarendon Press, Oxford, England, 1954).

2 Morse and Feshbach, Methods of Theoretical Physics (McGrawHill Book Company, Inc., New York, 1953).
}

such problems orthogonality and completeness theorems have not been established and therefore rigorous expansion methods do not exist. We will show that if the normal mode concept is retraced from its origins it is possible to establish a completely general orthonormality condition [Eq. (2.13)] valid for all continua without dissipation, irrespective of the order of the operators and the nature of the boundary conditions involved, as long as these are subject to the laws of mechanics.

In addition, the principle of virtual work always provides a means of decomposing any kind of disturbance into a spectrum of generalized forces. This Lagrangian approach provides a flexible formulation of the arbitrary source problem which is again valid in all cases. Clearly, mixed problems involving several types of degrees of freedom such as may occur in electroacoustics would be most simply treated by this method.

In principle the usefulness of the normal mode method is generally recognized, since, as already pointed out, it is a standard tool in field theory. But in fact it has never been extended to cover the theory of mechanical waves in unlimited or partially limited media. In part this is probably because one has not appreciated the real power of the method which is due to the combined generality of the orthonormality condition (2.13) and the flexibility of the Lagrangian method of formulating arbitrary sources. But in part this must also be ascribed to the role played by linear operator and function-space theory in the formal development of quantum mechanics, which for many years had unavoidably dominated the scene in mathematical physics. 
At this point one may remark that the use of normal modes as generalized coordinates is essentially a Hilbert space formulation, and he might well inquire how this technique is related to standard Hilbert space methods, such as those used by Marcuvitz $z^{3}$ in his work on diffraction and discussed in various textbooks (Friedman, ${ }^{4}$ Riesz and Nagy ${ }^{5}$.

The answer to this is of course that the space of normal coordinates is related to other Hilbert spaces by a linear transformation. This is their formal connection. Physically, the essential difference between them can be explained as follows.

In the normal coordinate space the representation is physical rather than formal, since it utilizes only the actual modes of vibration of a system; i.e., free modes that can always be observed and realized as standing waves. The progressive waves are then obtained by a summation of all transient modes excited by the source. The amplitude of each mode is calculated directly from the corresponding generalized force component due to the source and applied to this mode. The Sommerfeld radiation condition is satisfied automatically and does not appear in the formulation. In contradistinction to this, other Hilbert space representations utilize orthonormal systems which are formal and which often do not have a direct correspondence to the actual modes of vibration. Take for instance the well-known Sommerfeld integral representation of a simple harmonic point source in an infinite homogeneous space. It is essentially a Fourier-Bessel expansion of the source into an orthonormal family of functions representing radiation into a half-space. The modes associated with this representation are not physical modes of vibration. Thus they include exponential modes which are obviously not free modes, but require an excitation. They correspond to what Sommerfeld ${ }^{6}$ has called, in a different context, "singular eigenfunctions." The formal nature of these modes is also illustrated by their well-known but very odd correspondence to complex angles of incidence, which from the physicist's standpoint are, of course, a pure formalism devoid of meaning. In general this standard formalism is equivalent to the expansion of the source into an orthonormal distribution along a coordinate surface leading to a representation of the Green's function for a harmonic point source which is generally different on each side of the coordinate surface. Such a method obviously depends on the separation of coordinates and the formal spectral properties of the associated differential operators. The Green's function is also required to satisfy the Sommerfeld radiation condition. The transient is then obtained by a method of Laplace or Fourier transforms, i.e., by

\footnotetext{
${ }^{3}$ N. Marcuvitz, Commun. Pure Appl. Math. 4, 263 (1951).

${ }_{4} \mathrm{~B}$. Friedman, Principles and Techniques of Applied Mathematics (John Wiley and Sons, Inc., New York, 1956).

${ }^{5}$ F. Riesz and B. S. Nagy, Leçons d'Analyse Fonctionelle (Gauthiers-Villars, Paris, 1955).

${ }^{6}$ A. Sommerfeld, Partial Differential Equations in Physics (Academic Press, Inc., New York, 1949).
}

an integration over all frequency components of the source. Thus, although the method of normal coordinates is intimately related to the standard Hilbert space methods, it is not the same. The fact that the normal coordinates correspond to physical modes of vibration has the advantageous consequence that one may use the Lagrangian or variational technique of formulating the source by the principle of virtual work.

It might be well to point out another essential difference between the Green's function method and the present normal coordinates approach, which becomes apparent if the source is a force applied to the boundary. In the Green's function method all components of the solution satisfy the boundary conditions that the stresses have the required value corresponding to the exciting forces. This is not the case in the normal coordinates method if the exciting force is applied to a free boundary since all components of the solution are free modes with vanishing forces at that boundary. This is an important distinction from the formal mathematical viewpoint as well as the practical one.

It must be emphasized that this is essentially an elementary technique. Its development can proceed solely from the simple laws of mechanics, without any reference to the somewhat formidable mathematical apparatus of function spaces. Such a development is, within the confines of mechanics, entirely rigorous and above reproach. This technique is quite analogous to the Hamiltonian methods of classical and quantum field theory as presented for example by Heitler ${ }^{1}$ in his book The Quantum Theory of Radiation. It is significant that in this book, the author entirely avoids mentioning Hilbert spaces.

Insofar as simple harmonic source problems in separable coordinate systems are concerned, the method of normal coordinates claims no particular advantages over the linear operator and so-called $\delta$ function technique used by Marcuvitz and others. However, it will be noted in Sec. 2 that it leads in a natural and straightforward fashion to a formalism which in principle includes nonseparable systems, although no actual examples of such problems will be discussed in the present paper. In the usual separable coordinate systems it is most useful in the case of transient excitation, although here again in simple problems the Laplace-transform method is just as good. The simplicity and conciseness of the method of normal coordinates begins to bear fruit in the diffraction problem of Sec. 5, where we feel that the treatment compares advantageously with Garnir's ${ }^{7}$ longer and more complicated derivation by the Laplace transform method. This paper is intended only as an introduction to a method, which will be more fully illustrated in subsequent publications. As such, it only provides a glimpse of the method's versatility and general usefulness.

${ }^{7}$ H. G. Garnir, Bull. soc. roy. sci. Liege 3, 119 (1952); 3, 207 (1952); 8, 328 (1952). 
The first few sections are introductory in nature. They provide the foundation upon which future examples of the method will be built. Since the method is essentially a new one in the context of infinite mechanical media, and since the mathematical techniques, although simple, may in some respects be unfamiliar to the reader, it was deemed advisable to dwell at some length upon the basic features of the method before proceeding to the solution of an actual problem. In Sec. 2 we will give a brief summary of the principles of the method, and an orthonormality (diagonalization) condition valid in all cases will be deduced. In Sec. 3 we shall show how the principle of virtual work is used for formulating arbitrary sources, with special reference to the point source in acoustics and elasticity. In particular we will obtain the general formulation for an idealized explosive source, corresponding to the instantaneous injection of a unit volume at a point. In Sec. 4 we will discuss briefly this source in an infinite homogeneous medium, show the type of pressure wave associated with it and derive several alternate representations thereof. In Sec. 5 we will solve the problem of an explosive point source of this type in a fluid wedge or corner with rigid boundaries, and show that this method leads to an explicit closed formula in terms of elementary functions. The simplicity of this problem, when formulated by the normal coordinates method, gives a clear picture of the technique and of some of its advantages. Other applications have been discussed elsewhere. ${ }^{8}$

\section{SUMMARY AND FOUNDATIONS OF THE METHOD}

In the theory of small, undamped, oscillations of holonomic systems of particles with $n$ degrees of freedom, the potential and kinetic energies $V, T$ are positive definite quadratic forms of the particle coordinates $\gamma_{i}$ and velocities $\dot{\gamma}_{i}$ in configuration space. In other words if $V$ and $T$ are represented by symmetric matrices $\mathbf{V}, \mathbf{T}$ and the state of the system by the position and velocity vectors $\gamma, \dot{\gamma}$ one has

$$
\begin{aligned}
& V=\frac{1}{2} \gamma^{\prime} \bigvee_{\boldsymbol{\gamma}} \\
& T=\frac{1}{2} \dot{\gamma}^{\prime} \mathbf{T} \dot{\gamma},
\end{aligned}
$$

where $\boldsymbol{\gamma}^{\prime}$ is the transpose of $\boldsymbol{\gamma}$. Direct application of Lagrange's equations in this configuration space yields a system of $n$-coupled equations of motion for the free oscillations

$$
\mathbf{T} \ddot{\gamma}+V_{\boldsymbol{\gamma}}=0 .
$$

One may assume that, except for an arbitrary multiplying constant, the solutions are of the type,

$$
\gamma=q \mathbf{a}
$$

where $q$ is a function of $t$ only and a fixed vector. The assumption of simple harmonic motion leads to an

\footnotetext{
${ }^{8}$ M. A. Biot and I. Tolstoy, Shell Development Company, EPR Report, "The Hilbert space method in wave propagation and diffraction for arbitrary sources" (October, 1955).
}

eigenvalue problem and the determination of eigenfrequencies $\omega_{k}$ and eigenvectors $\mathbf{a}_{k}$. The method of normal modes consists of taking the $q_{k}$ as the new coordinates. This is done by means of the linear transformation,

$$
\boldsymbol{\gamma}=\mathbf{A q}
$$

A being the square matrix composed of eigenvector columns $\mathbf{a}_{k}$ and $\mathbf{q}$ the column matrix of the $q_{k}$. This is a principal axis transformation which simultaneously diagonalizes the two quadratic forms $V, T$

$$
\begin{aligned}
& \mathbf{A}^{\prime} \mathbf{V A}=\boldsymbol{\lambda} \mathfrak{u} \\
& \mathbf{A}^{\prime} \mathbf{T A}=\mathfrak{u}
\end{aligned}
$$

$\lambda, \boldsymbol{u}$ being diagonal matrices of elements

$$
\begin{aligned}
\lambda_{k} & =\omega_{k}^{2} \\
\sum_{i, j} T_{i j} a_{\imath k} a_{j l} & =\delta_{k \imath} \mu_{k l}=\delta_{k l} \mu_{k} .
\end{aligned}
$$

Equation (2.9) expresses in a space of metric tensor $T_{i j}$ the fact that the eigenvectors are orthogonal. If the eigenvectors $\mathbf{a}_{k}$ in Eq. (2.4) are multiplied by $\mu_{k}{ }^{-\frac{1}{2}}$ (normalization), (2.5) transforms $\mathbf{T}$ into the unit matrix. However, we prefer to leave the eigenvectors unnormalized. It is then seen that the equations of forced motion are

$$
\ddot{q}_{k}+\omega_{k}^{2} q_{k}=Q_{k} \mu_{k}{ }^{-1},
$$

where the $Q_{k}$ represent generalized forces obtained from the unnormalized set of eigenvectors by the principle of virtual work.

These well-known results are readily transposed to the case of continuous systems. The energies (2.1) and (2.2) are replaced by energy densities, defining a Lagrangian density. Substituting this into Lagrange's equations for continuous media one obtains the equations of motion in partial differential form. In hydrodynamics and elasticity the assumption of small motion leads to various forms of the wave equation or generalizations thereof. For example we may consider the general equations for wave propagation in a fluid which is not homogeneous and which is initially at rest and in equilibrium under the action of a body force. This is a particular case of the general theory of wave propagation in a prestressed medium which was developed by Biot. ${ }^{9}$ The initial stress distribution in the present case reduces to a hydrostatic pressure $P$. If $\boldsymbol{\gamma}$ is the particle displacement, $\lambda(x, y, z)$ the bulk modulus and $\rho(x, y, z)$ the density of the fluid, the general equations become

$$
\rho \ddot{\gamma}_{2}-\frac{\partial}{\partial x_{i}}(\lambda \nabla \cdot \gamma)+\sum^{j} \frac{\partial \gamma_{j}}{\partial x_{i}} \frac{\partial P}{\partial x_{j}}-\frac{\partial P}{\partial x_{i}}(\nabla \cdot \gamma)=0,
$$

which is an analog of Eq. (2.3). Simple harmonic solutions satisfying the boundary conditions define

\footnotetext{
${ }^{9}$ M. A. Biot, J. Appl. Phys. 11, 522-530 (1940).
} 
an infinite spectrum of eigenfrequencies $\omega_{k}$ and eigenvectors $\mathbf{a}_{k}$. In the case of an enclosure this spectrum is in general infinite and discrete, and the linear transformation

$$
\boldsymbol{\gamma}=\sum_{k=0}^{\infty} q_{k} \mathbf{a}_{k}
$$

now defines a rotation in Hilbert space. In the general case of a three-dimensional enclosure this will be a multiple series over several indices.

The orthonormality condition (2.9) rewritten for the continuum is

$$
\int_{\tau} \rho \mathbf{a}_{k} \cdot \mathbf{a}_{l} d \tau=\mu_{k l} \delta_{k l}
$$

where integration extends over the whole medium. This condition is completely general and is valid regardless of whether the problem can be described by familiar types of operator such as (2.11) or not. In writing it, the only assumption made is the rather trivial one claiming that it is always possible to transfer the equations of discrete systems to bounded enclosures. It is not even required that the problem be separable. In other words $E q$. (2.13) is the general orthonormality condition for all conservative mechanical systems, and for any boundary conditions providing these too are conservative. It is seen from the preceeding that it owes its nature to the principles of mechanics which provide sufficient justification for its use in all cases.

In the special case of Eq. (2.11), under simple harmonic conditions and in separable coordinate systems, this equation separates into Sturm-Liouville operators. Equation (2.13) also separates into several equations, one of which is then the standard form of the orthonormality condition in view of the weighting factor of the first term in Eq. (2.11). The necessity of a weighting factor in the orthonormality integral for certain Sturm-Liouville problems may thus be viewed as a consequence of the non-Cartesian nature of the configuration metric. Equation (2.13) can be said to describe the diagonalization of an infinite kinetic energy matrix.

The equations of motion for the $q_{k}$ still have the form (2.10).

As evidenced by our continued use of subscripts, we have assumed so far that the spectrum is discrete, i.e., that we have been dealing with enclosures. Actually this need not be so, and we may drop all subscripts and replace the summation of Eq. (2.12) by an integral, which in the case of several dimensions may be double or triple depending upon the coordinate system. This process is beset with one rather minor difficulty with which we shall now concern ourselves. When the medium is unbounded and the spectrum is continuous, the integral in Eq. (2.13), which we may for the time being assume to be simple rather than multiple, will usually diverge. This feature may be handled in several ways.
One can introduce the concept of mode density, i.e., of the number of oscillators in a band $\omega, \omega+d \omega$. Or one may appeal to a modified form of the orthonormality conditions introduced by Weyl ${ }^{6}$ and commonly used in quantum theory. We have decided to use a less rigorous but more compact and fundamentally equivalent symbolism encountered sometimes in the formulation of Green's functions in infinite domains, ${ }^{6}$ or in passing from orthogonal series expansions to the corresponding integral representations. It consists of writing symbolic equations of the following type:

$$
\begin{aligned}
& \int_{-\infty}^{+\infty} \cos ^{2} \beta z d z=\int_{-\infty}^{+\infty} \sin ^{2} \beta z d z=\frac{\pi}{d \beta} \\
& \int_{0}^{\infty} J_{0}{ }^{2}(\kappa r) r d r=\int_{0}^{\infty} J_{1}{ }^{2}(\kappa r) r d r=\frac{1}{\kappa d \kappa} \\
& \int_{0}^{\infty} J_{\nu}{ }^{2}(\kappa r) r d r=\frac{1}{\kappa d \kappa} .
\end{aligned}
$$

Relations of this kind may always be found and justified in particular cases by examining the asymptotic behavior of the eigenvalues of an enclosure which is being expanded. For example, if one were to consider a string fixed at both ends, of length $2 l$, extending from $z=-l$ to $z=+l$, the eigenfunctions are of the form $\sin \beta_{n} z, \beta_{n}=n \pi / l$, and

$$
\int_{-l}^{+l} \cos ^{2} \beta_{n} z d z=l .
$$

The difference between neighboring eigenvalues is

$$
\Delta \beta=\beta_{n+1}-\beta_{n}=\pi / l .
$$

At the limit $l \rightarrow \infty$, we write $l=\pi / \Delta \beta$ and Eq. (2.14) follows. On the other hand, Eqs. (2.15) can be deduced by considering a circular membrane and passing to the limit of infinite radius, and in exactly similar fashion we may start with a membrane occupying a circular sector and obtain Eq. (2.16) for wedge-shaped spaces.

The formalism for passing to unbounded spaces is now clear. The normalization factor $\mu_{k}$ is defined by Eqs. (2.13) and (2.14) or Eqs. (2.15) and (2.16), and the $q_{k}$ in Eq. (2.10) are now differentials of the first or higher orders. Equation (2.12) then gives the solution in the form of a simple or multiple integral. This procedure is clearly equivalent to obtaining first the solution for a suitable enclosure, and then expanding it in one or more directions by means of symbolic equations of the type (2.14), (2.15), and (2.16).

Except for the formulation of $Q_{k}$, we are now in possession of all the results that we shall need. The $Q_{k}$ depend upon the type of problem and the sources that one assumes. In the next section we have formulated $Q_{k}$ with special attention to the point source problem in acoustics and elasticity. 


\section{THE GENERALIZED FORCE REPRESENTATION OF ARBITRARY SOURCES}

Let $\gamma$ represent the particle displacement in a fluid or elastic medium, $\mathbf{F}$ being an applied force per unit volume. The component of $\boldsymbol{\gamma}$ corresponding to the $k$ th mode according to $\mathrm{Eq}$. (2.12)

$$
\gamma_{k}=q_{k} \mathbf{a}_{k}
$$

The principle of virtual work enables one to decompose F into its Hilbert space components $Q_{k}$ from the knowledge of its scalar product with one eigenvector instead of going through the complete transformation (2.12). A variation $\delta q_{k}$ is associated with a virtual displacement

$$
\delta \gamma_{k}=\mathbf{a}_{k} \delta q_{k}
$$

of the medium. The virtual work is represented by the following scalar

$$
Q_{k} \delta q_{k}=\int_{\tau} \mathbf{F} \cdot \delta \gamma_{k} d \tau,
$$

where the integration extends over the whole volume in which $\mathbf{F}$ is effective. We have therefore

$$
Q_{k}=\int_{\tau} \mathbf{F} \cdot \mathbf{a}_{k} d \tau .
$$

This is the most general form of $Q_{k}$ in which $\mathbf{F}$ is an arbitrary function of $x, y, z, t$, e.g., a moving distributed transient. In the case of applied stresses $f$ per unit surface,

$$
Q_{k}=\int_{s} \mathbf{f} \cdot \mathbf{a}_{k} d s .
$$

Such would be the representation for a plane of shear in an elastic solid or of forces distributed along a boundary of the medium.

- Of particular interest for many propagation and diffraction problems in acoustics and elasticity are concentrated sources, e.g., point sources. As an example we now derive $Q_{k}$ in its general form for the compressional point source.

Consider a spherical portion of the medium, of volume $V$ centered at $r=0$ (Fig. 1), which has been kept in a state of constant compression from time $t=-\infty$ to $t=0$. At $t=0$ the constraining forces are removed. This assumes on the surface of the sphere a stress

where

$$
\mathbf{f}=-\boldsymbol{\sigma} \mathbf{1}(-t)
$$

$$
\begin{aligned}
1(-t) & =1, & t<0 \\
& =0, & t>0 .
\end{aligned}
$$

Equation (3.5) gives the integral over the surface $s$

$$
\begin{aligned}
Q_{k} & =-1(-t) \int_{s} \sigma \mathbf{a}_{k} \cdot d \mathbf{s} \\
& =-1(-t) \sigma \int_{V} \nabla \cdot \mathbf{a}_{k} d V
\end{aligned}
$$

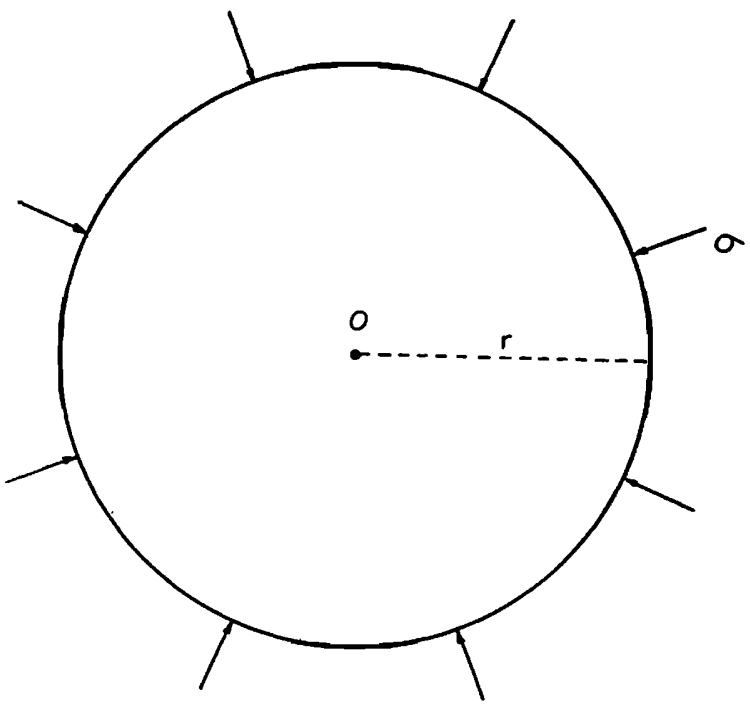

FIG. 1. Compressed sphere subject to surface stress $\sigma$. As $r \rightarrow 0$ and the volume $V \rightarrow 0$ this becomes a point source.

For small $V$

$$
Q_{k}=-1(-t) \sigma V\left(\nabla \cdot \mathbf{a}_{k}\right)_{r=0} .
$$

$\sigma V$ is thus a measure of the intensity of the source, while $\left(\nabla \cdot \mathbf{a}_{k}\right)_{r=0}$ is a measure of its influence on each mode. If $\epsilon$ is the volume change per unit volume we have

$$
\lambda \epsilon=\sigma,
$$

where $\lambda$ is the bulk modulus. Hence

$$
\lambda \epsilon V=\sigma V \text {. }
$$

Now $\epsilon V$ represents the total incremental change in volume. Therefore this type of source corresponds to an injection of volume $\epsilon V$; i.e., it simulates an instantaneous explosion. For a unit volume injection, $\epsilon V=1$, we have

$$
Q_{k}=-1(-t) \lambda\left(\nabla \cdot \mathbf{a}_{k}\right)_{r=0} .
$$

We note for future reference that if we write in this case

$$
C_{k}=-\mu_{k}^{-1} \lambda\left(\nabla \cdot \mathbf{a}_{k}\right)_{r=0} .
$$

We may write the solutions of Eqs. (2.10) for $t>0$ as

$$
q_{k}=C_{k} \frac{\cos \omega_{k} t}{\omega_{k}^{2}} .
$$

Finally it is clear that for arbitrary time dependence $\psi(t)$ of the compressional point source

$$
Q_{k}=-\psi(t) \lambda\left(\nabla \cdot \mathbf{a}_{k}\right)_{r=0} .
$$

It should be noted that the generalized form for other types of sources are just as easy to obtain. For instance an instantaneous torque concentrated in the vicinity of a point in a solid would lead to generalized forces proportional to $\nabla \times \mathbf{a}_{k}$ at this point. 


\section{THE INSTANTANEOUS EXPLOSION IN VARIOUS COORDINATE SYSTEMS}

We will now derive a few integral representations for the waves due to the instantaneous unit volume injection point source in an infinite homogeneous fluid. In so doing we will be able to verify that this type of source leads to a spherically propagating pressure doublet (i.e., an infinite compression followed instantaneously by an infinite rarefaction) and to illustrate the basic methematical manipulations in their simplest form.

We begin with a cylindrical coordinate system $r, z, \theta$ in an infinite homogeneous fluid of sound velocity $\alpha$, density $\rho$. We assume a source of the aforementioned type located at the origin. The excited modes will be symmetrical with respect to the polar axis and the plane $z=0$. The spectrum is continuous in $r, z$ and each mode will correspond to a second order differential as explained in Sec. 2. In the present case we are dealing with irrotational motion ( $\rho$ is constant) and it is convenient to introduce hydrodynamic potentials. We therefore write, dropping subscripts

$$
\begin{aligned}
d^{2} \varphi & =d^{2} q \cos \beta z J_{0}(\kappa r)=\psi d^{2} q, \\
\omega & =\alpha\left(\beta^{2}+\kappa^{2}\right)^{\frac{3}{3}},
\end{aligned}
$$

where $\varphi, \psi$ may be taken as displacement potentials obeying the equation

$$
\nabla^{2} \varphi+\omega^{2} \varphi / \alpha^{2}=0
$$

and are related to the acoustical pressure $p$ and displacements $\boldsymbol{\gamma}$, a as follows

$$
\begin{aligned}
& p=-\rho \frac{\partial^{2} \varphi}{\partial t^{2}} \\
& \gamma=\nabla \varphi \\
& \mathbf{a}=\nabla \psi .
\end{aligned}
$$

Since $d^{2} q$ will be given by Eq. (3.14) (with no subscripts), the complete solution will have the forms

$$
\gamma=\iint \mathrm{a} d^{2} q
$$

or

$$
\varphi=\iint d^{2} \varphi
$$

Explicitly, Eq. (2.13) defines $\mu$ as

$$
\begin{aligned}
\mu & =\int_{\tau} \rho \mathbf{a}^{2} d \tau=2 \pi \rho \int_{-\infty}^{+\infty} d z \int_{0}^{\infty}(\nabla \psi)^{2} r d r \\
& =2 \pi \rho \int_{-\infty}^{+\infty} d z \int_{0}^{\infty}\left[\beta^{2} \sin ^{2} \beta z J_{0}^{2}(\kappa r)\right.
\end{aligned}
$$

$$
\left.+\kappa^{2} \cos ^{2} \beta z J_{1}^{2}(\kappa r)\right] r d r \text {. }
$$

Using Eqs. (2.14), (2.15), and (4.2)

$$
\mu=\rho-\frac{\omega^{2}}{\alpha^{2}} \frac{2 \pi^{2}}{\kappa d \kappa d \beta} .
$$

Since $\lambda=\rho \alpha^{2}$, Eqs. (3.13) and (3.14) give

$$
d^{2} q=-\frac{1}{2 \pi^{2}} \frac{\alpha^{4}}{\omega^{2}}(\nabla \cdot \mathbf{a})_{r=\boldsymbol{z}=0} \frac{\cos \omega t}{\omega^{2}} \kappa d \kappa d \beta,
$$

and noting that

$$
\nabla \cdot \mathbf{a}=\nabla^{2} \psi=-\omega^{2} \psi / \alpha^{2},
$$

we have, by Eq. (4.1),

$$
d^{2} q=\frac{\alpha^{2}}{2 \pi^{2}} \frac{\cos \omega t}{\omega^{2}} \kappa d \kappa d \beta .
$$

Substituting into Eqs. (4.1) and (4.8) the spherical wave diverging from the source is represented by the displacement potential

$$
\varphi=\frac{\alpha^{2}}{2 \pi^{2}} \int_{0}^{\infty} \cos \beta z d \beta \int_{0}^{\infty} J_{0}(\kappa r) \frac{\cos \omega t}{\omega^{2}} \kappa d \kappa .
$$

This may be integrated with the help of familiar transforms, ${ }^{10}$ with the result

$$
\begin{gathered}
\varphi=-\frac{1}{4 \pi} \cdot \frac{1}{R} 1\left(t-\frac{R}{\alpha}\right), \quad t>0 \\
R=\left(r^{2}+z^{2}\right)^{\frac{1}{2}} .
\end{gathered}
$$

The pressure wave has therefore the form

$$
p=\frac{\rho}{4 \pi} \cdot \frac{1}{R} \delta^{\prime}\left(t-\frac{R}{\alpha}\right), \quad t>0 .
$$

Where $\delta^{\prime}$ is the "derivative" of the Dirac delta function

$$
\delta^{\prime}(x)=\frac{d}{d x} \delta(x),
$$

$p$ has thus the character of an instantaneous doublet: it starts with an infinite compression, followed instantly by an infinite rarefaction.

Other representations of the spherical transient (4.16) may be obtained by going to other coordinate systems. For example, in a Cartesian system $x, y, z$ we would have

$$
\begin{aligned}
\varphi & =-\frac{1}{4 \pi} \frac{1}{R} 1\left(t-\frac{R}{\alpha}\right) \\
& =\frac{\alpha^{2}}{\pi^{3}} \int_{0}^{\infty} \int_{0}^{\infty} \int_{0}^{\infty} \cos \beta z \cos \gamma y \cos \kappa x \frac{\cos \omega t}{\omega^{2}} d \beta d \gamma d \kappa \\
\omega & =\alpha\left(\beta^{2}+\gamma^{2}+\kappa^{2}\right)^{\frac{3}{2}} .
\end{aligned}
$$

${ }^{10}$ The Bateman project staff, A. Erdelyi, editor, Tables of Integral Transforms (McGraw-Hill Book Company, Inc., New York, 1954), Vols. I and II. See also reference 1. 
An equivalent form, similar to some results we will obtain in sec. 5 , may be arrived at by considering a cylindrical system $r, z, \theta$, with the source situated off the axis at $z=0, \theta=0, r=r_{0}$ (Fig. 2). The excited modes are

$$
\begin{aligned}
d^{2} \varphi_{n} & =d^{2} q_{n} \cos n \theta J_{n}(\kappa r) \cos \beta z=d^{2} q_{n} \psi_{n} \\
\omega & =\alpha\left(\beta^{2}+\kappa^{2}\right)^{\frac{1}{2}} .
\end{aligned}
$$

Here $n$ is an integer: the $\theta$ spectrum is discrete in contradistinction to the continuous $r, z$ spectrum and will be associated with a summation.

Equations (2.13), (2.14), and (2.16) give the normalizing coefficient

$$
\begin{aligned}
\mu_{n} & =\rho \int_{0}^{2 \pi} d \theta \int_{-\infty}^{+\infty} d z \int_{0}^{\infty}\left(\nabla \psi_{n}\right)^{2} r d r \\
& =\rho \frac{\omega^{2}}{\alpha^{2}} \cdot \frac{\pi^{2}}{\kappa d \kappa d \beta} .
\end{aligned}
$$

We may substitute in Eqs. (3.13) and (3.14) and use Eqs. (4.13) and (4.8) with the result

$$
\begin{aligned}
\varphi=- & \frac{1}{4 \pi} \cdot \frac{1}{R} 1\left(t-\frac{R}{\alpha}\right)=\frac{\alpha^{2}}{\pi^{2}} \sum_{n=0}^{\infty} \cos n \theta . \\
& \times \int_{0}^{\infty} \int_{0}^{\infty} J_{n}(\kappa r) J_{n}\left(\kappa r_{0}\right) \cos \beta z \frac{\cos \omega t}{\omega^{2}} \kappa d \kappa d \beta .
\end{aligned}
$$

The identity of the two sides in Eq. (4.22) clearly follows from the fact that we are merely expressing the transient (4.16) in a different coordinate system. Actually this result may be verified directly by evaluating the double integral with the help of known transforms such as Macdonald's integral (see Sec. 5).

At this point several remarks must be made. First, it is clear that we have obtained directly the progressive transient wave, without requiring intermediate simple harmonic solutions: this is a general and characteristic feature of this method. Secondly, the solution for any other time dependence of the source can be obtained by applying Duhamel's theorem to the solution for the instantaneous explosion. This would be the proper and logical procedure for securing the progressive solution for a point source of time dependence $e^{i \omega_{0} t}$. In the simple case under discussion this would lead, for $\partial \varphi / \partial t$, to the somewhat trivial equation

$$
\begin{aligned}
\frac{\partial \varphi}{\partial t}=-\frac{1}{4 \pi} \int_{-\infty}^{t} \frac{e^{i \omega_{0} \tau} \delta[(t-\tau)-(R / \alpha)]}{R} d \tau \\
=-\frac{1}{4 \pi} \cdot \frac{1}{R} e^{i \omega_{0}[t-(R / \alpha)]} .
\end{aligned}
$$

But if one were to write $\psi(t)=e^{i \omega_{0} t}$ in Eq. (3.15), one would obtain an altogether different result. Indeed this result would not represent a progressive wave, but

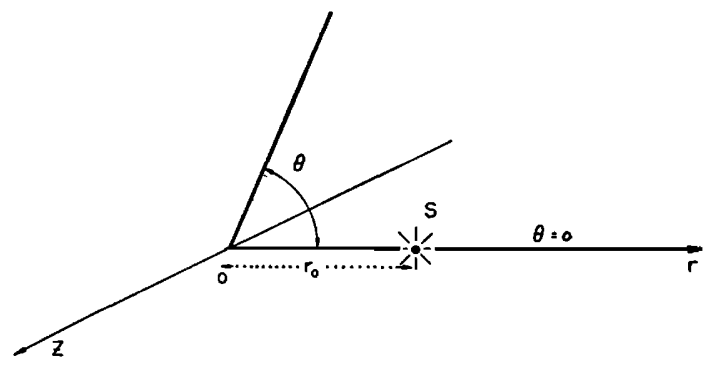

Fig. 2. Cylindrical coordinate system with source $S$ off axis at $z=0, \theta=0, r=r_{0}$.

could be forced to do so by a change in contour. It would then be equivalent to Sommerfeld's famous integral. Lack of space forbids us to discuss this rather subtle point more thoroughly, but we may note this much in passing: by substituting $\psi(t)=e^{i \omega_{0} t}$ in Eq. (3.15) one cannot really expect to obtain directly a progressive solution, for this type of time dependence implies a source that has been acting for all time, and the solution should include reflections from the enclosure walls, even after these have been removed to infinity. The procedure typified by Eq. (4.23) on the other hand, will yield a progressive wave because it corresponds to putting an $e^{i \omega_{0} t}$ source starting at $t=t_{0}$ into an infinite space, and gradually removing $t_{0}$ to $-\infty$. A more complete discussion of these features will be found in reference 3 .

\section{THE INSTANTANEOUS EXPLOSION : DIFFRACTION BY A WEDGE OR CORNER}

We will now solve the problem of the diffraction of a spherical pulse by an infinite plane wedge or corner. The diffraction by wedges has been the subject of a great many theoretical investigations. Sommerfeld ${ }^{11}$ solved the acoustical problem for plane transients, and Friedlander ${ }^{12}$ worked out in detail this case for certain specific pulse shapes. Pauli ${ }^{13}$ and others extended results of this type to electromagnetic waves and conducting wedges. The simple harmonic point source in a wedge was worked out by Oberhettinger. ${ }^{14}$ Asymptotic solutions have been examined by Keller, Lewis, and Seckler. ${ }^{15}$ Keller and Blank ${ }^{16}$ have also given a solution of the plane transient case, using Busemann's method of conical flow. An exhaustive bibliography will be found in a review article by Bouwkamp. ${ }^{17}$ Until recently no solution of the transient point source problem had been published. We will show that the Hilbert space method leads easily to an explicit solution in closed form, involving only elementary functions. A solution

\footnotetext{
11 A. Sommerfeld, Math. Ann. 45, 263 (1894) ; 45, 317 (1896)

12 F. G. Friedlander, Proc. Roy. Soc. (London) A186, 322, 352 (1946).

${ }_{13}$ W. Pauli, Phys. Rev. 54, 924 (1938).

${ }_{14} \mathrm{~F}$. Oberhettinger, Commun. Pure Appl. Math. 7, 551 (1954).

${ }^{15}$ Keller, Lewis, and Seckler, Commun. Pure Appl. Math. 9, 207 (1956).

16 J. B. Keller and A. Blank, Commun. Pure Appl. Math. 4, 75 (1951).

${ }_{17}$ C. J. Bouwkamp, Repts. Progr. in Phys. 17, 35 (1954).
} 
equivalent to ours has been published in a series of little known articles by Garnir. ${ }^{7}$ His method is radically different, since he uses Heaviside's operational approach, as adapted to wave theory by Cagniard ${ }^{18}$ and Pekeris. ${ }^{19}$ A comparison of his lengthy derivation with ours illustrates the relative directness and simplicity of the normal coordinate approach. It is not only simpler and elegant, but also much more flexible. It leads naturally to new forms of the solution (e.g., a useful convergent series). In addition, we have reached some interesting physical conclusions so that this choice of an example not only provides a simple illustration of some advantages of the present approach, but also represents a contribution to diffraction theory per se.

Consider an infinite wedge or corner bounded by rigid planes $\theta=0, \theta=\zeta$ intersecting along the $z$ axis (apex). We assume the region $0<\theta<\zeta$ to be filled with homogeneous fluid (Fig. 3). We may call this a wedge if $\zeta>\pi$, a corner if $\zeta<\pi$. For a source located at $r=r_{0}$, $\theta=\theta_{0}, z=0$ the excited modes are

$$
\begin{aligned}
d^{2} \varphi_{n} & =d^{2} q_{n} \cos \frac{n \pi}{\zeta}-\theta J_{n \pi / 5}(\kappa r) \cos \beta z=d^{2} q_{n} \psi_{n} \\
\omega & =\alpha\left(\beta^{2}+\kappa^{2}\right)^{\frac{1}{2}} .
\end{aligned}
$$

Equation (2.13) gives

$$
\mu_{n}=\rho \int_{0}^{\zeta} d \theta \int_{-\infty}^{+\infty} d z \int_{0}^{\infty}\left(\nabla \psi_{n}\right)^{2} r d r
$$

and by virtue of Eqs. (2.16) and (2.14)

$$
\mu_{n}=\frac{1}{2} \zeta \frac{\omega^{2}}{\alpha^{2}} \frac{\pi}{\kappa d \kappa d \beta} .
$$

Substituting in Eqs. (3.13), (3.14), and (5.1)

$$
\begin{aligned}
\varphi= & \frac{2 \alpha^{2}}{\pi \zeta} \sum_{n=0}^{\infty} \cos \frac{n \pi}{\zeta} \theta \cos \frac{n \pi}{\zeta} \theta_{0} \\
& \times \int_{0}^{\infty} \int_{0}^{\infty} J_{n \pi / \zeta}(\kappa r) J_{n \pi / \zeta}\left(\kappa r_{0}\right) \cos \beta z \frac{\cos \omega t}{\omega^{2}} \kappa d \kappa d \beta .
\end{aligned}
$$

If $\zeta=\pi / m, m$ being an integer, it is easily shown that this result can be represented by a sum of forms such as Eq. (4.22), and that the solution is then equivalent to the original source plus $2 m-1$ mirror images distributed on a circle of radius $r_{0}$. But this is a somewhat trivial case, for it is well known that under these circumstances the complete solution is given by such a distribution of sources - there are no diffracted terms. ${ }^{6,20}$

We assume therefore $\zeta=\pi / \nu, \nu$ not an integer.

${ }^{18} \mathrm{~L}$. Cagniard, Reflexion et refraction des ondes seismiques progressives (Gauthiers-Villars, Paris, 1939).

${ }^{19}$ C. L. Pekeris, Proc. Natl. Acad. Sci. 41, 469, 629 (1955).

20 J. B. Keller, Commun. Pure Appl. Math, 6, 505 (1953).
Note that we may expect the solution to consist of two types of terms: the images on one hand, and a diffracted wave on the other. On simple geometrical grounds (Fig. 4) it is seen that the diffracted wave will always arrive at a later time than the latest image contribution. If we call $t_{0}$ the time of the first (direct) signal and $\tau_{0}$ the time of arrival of the leading edge of the diffracted wave (corresponding to the minimal source to apex to receiver path), we have

$$
\begin{aligned}
t_{0}= & \frac{1}{\alpha}\left(\left(r-r_{0}\right)^{2}+z^{2}\right)^{\frac{1}{2}}, \\
& \\
\tau_{0}= & \frac{1}{\alpha}\left(\left(r+r_{0}\right)^{2}+z^{2}\right)^{\frac{1}{2}} .
\end{aligned}
$$

To evaluate the integral in Eq. (5.4) we first differentiate with respect to $t$ and use the result ${ }^{10}$

$$
\begin{aligned}
\int_{0}^{\infty} \cos \beta z \frac{\sin \alpha t\left(\beta^{2}+\kappa^{2}\right)^{\frac{1}{3}}}{\left(\beta^{2}+\kappa^{2}\right)^{\frac{1}{3}}} & d \beta \\
& =\frac{\pi}{2} J_{0}\left[\kappa\left(\alpha^{2} t^{2}-z^{2}\right)^{\frac{1}{2}}\right], \quad \alpha t>z \\
& =0, \quad \alpha t<z .
\end{aligned}
$$

This gives

$$
\begin{aligned}
\frac{\partial \varphi}{\partial t} & =-\frac{\alpha}{\zeta} \sum_{n=0}^{\infty} \cos \frac{n \pi}{\zeta} \theta_{0} \cos \frac{n \pi}{\zeta} \rightarrow I_{n}, \quad \alpha t>z \\
& =0, \alpha t<z
\end{aligned}
$$

where

$$
I_{n}=\int_{0}^{\infty} J_{n \pi / \zeta}(\kappa r) J_{n \pi / \zeta}\left(\kappa r_{0}\right) J_{0}\left[\kappa\left(\alpha^{2} t^{2}-z^{2}\right)^{\frac{1}{1}}\right] \kappa d \kappa .
$$

This integral may be evaluated explicitly. It is a particular case of MacDonald's integral which will be

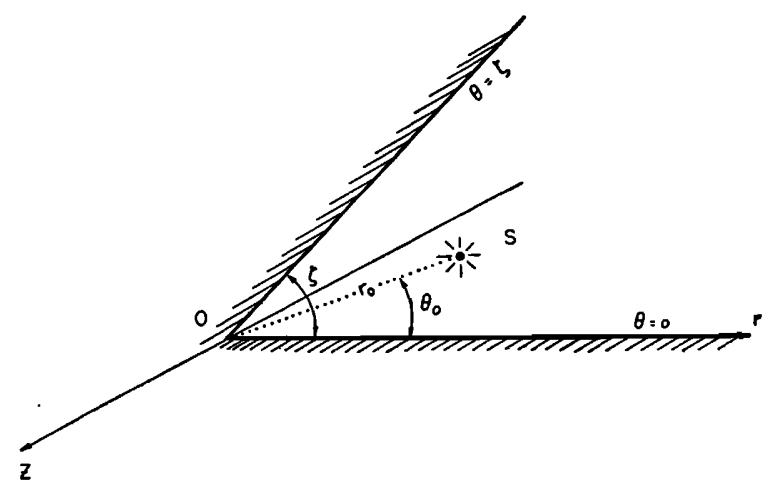

Fig. 3. Source $S$ at $r=r_{0}, \theta=\theta_{0}, z=0$ in a fluid filled corner of angle $\zeta$. 


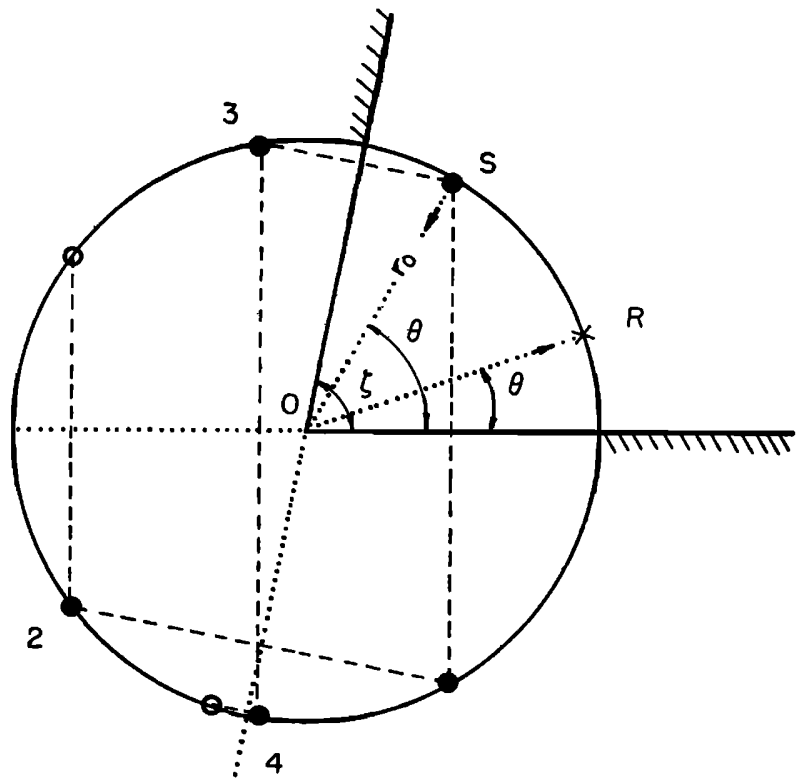

FIG. 4. Distribution of images for a corner of angle $\zeta$ slightly less than $\pi / 2$ and a source $S$ at $r=r_{0}$. The heavy black points are observable mirror images, whereas the white points are constructed as images but are not observable as such. When $\zeta \rightarrow \pi / 2$ images 2 and 4 coalesce, and the non-observable images coincide with the observable ones. With a receiver $R$ as shown $\left(r=r_{0}\right)$, it is clear that the path $S O R=2 r_{0}$ is larger than the distance of $R$ to any of the images. This is true no matter what the position of $R$, except if it is on the apex.

found discussed by Watson. ${ }^{21}$ The result is

$$
\begin{aligned}
& t<t_{0}, \quad I_{n}=0 \\
& t_{0}<t<\tau_{0}, \quad I_{n}=\frac{1}{\pi r r_{0} \sin \xi} \cos \frac{n \pi}{\zeta} \xi \\
& \xi=\operatorname{Arc~} \cos \frac{r^{2}+r_{0}^{2}+z^{2}-\alpha^{2} t^{2}}{2 r r_{0}}, \quad 0 \leqslant \xi \leqslant \pi \\
& \tau_{0}<t, \quad I_{n}=-\frac{1}{\pi r r_{0} \sinh \eta} \sin \frac{n \pi}{\zeta} \pi e^{-n \pi \eta / \zeta} \\
& \eta=\operatorname{Arg} \cosh \frac{\alpha^{2} t^{2}-\left(r^{2}+r_{0}{ }^{2}+z^{2}\right)}{2 r r_{0}}, \\
& 0 \leqslant \eta \leqslant \infty,
\end{aligned}
$$

where $t_{0}, \tau_{0}$ have been defined as in Eqs. (5.5) and (5.6). Clearly the form (5.11) corresponds to the image contributions:

$$
\frac{\partial \varphi}{\partial t}=-\frac{\alpha}{\pi \zeta r r_{0} \sin \xi} \sum_{n=0}^{\infty} \frac{n \pi}{\zeta} \frac{n \pi}{\zeta}-\theta_{0} \cos \frac{n \pi}{\zeta} \theta \cos \frac{n \pi}{\zeta} \xi .
$$

Each term of this series may be written as the sum of

${ }^{21}$ G. N. Watson, Theory of Bessel Functions (Cambridge University Press, New York, 1922). four cosine terms, and one will recognize the result to be

$$
\frac{\partial \varphi}{\partial t}=-\frac{\alpha}{4 \pi r r_{0} \sin \xi} \sum_{m}^{\infty} \delta\left(\xi \mp \theta \mp \theta_{0}-2 m \zeta\right),
$$

where the \pm signs are used to show that for each value of $m$ we have a sum of four terms corresponding to all possible combinations of these signs. There is a finite number of permissible values of $m$ determined by the condition $\xi \leq \pi$ [Eq. (5.12)] and corresponding to the feature that the number of observable images is always finite (Fig. 4). The distance between image and receiver is

$R=\left[r^{2}+r_{0}^{2}+z^{2}-2 r r_{0} \cos \left(2 m \zeta \pm \theta \pm \theta_{0}-\xi\right)\right]^{\xi}=\alpha t$

where $t$ is the corresponding arrival time. The Dirac delta function has the property

$$
\delta\left(\xi \mp \theta \mp \theta_{0}-2 m \zeta\right) d \xi=\delta\left(t-\frac{R_{m}}{\alpha}\right) d t
$$

and Eq. (5.12) shows that

Therefore

$$
r r_{0} \sin \xi d \xi=\alpha^{2} t d t .
$$

$$
\begin{aligned}
\frac{\alpha}{r r_{0} \sin \xi} \delta\left(\xi \mp \theta \mp \theta_{0}-2 m \zeta\right) \\
=\frac{1}{\alpha t} \delta\left(t-\frac{R_{m}}{\alpha}\right)=\frac{1}{R_{m}} \delta\left(t-\frac{R_{m}}{\alpha}\right)
\end{aligned}
$$

and

$$
\frac{\partial \varphi}{\partial t}=-\frac{1}{4 \pi} \sum_{m} \frac{1}{R_{m}} \delta\left(t-\frac{R_{m}}{\alpha}\right),
$$

where there will be in general four terms for each value of $m$, corresponding to all realizable sign combinations in Eq. (5.17). The acoustical pressure solution, obtained by applying the operator $-\rho \partial / \partial t$ to Eq. (5.21) is therefore equivalent to a number of pressure doublets [Eq. (4.17)] corresponding to all possible mirror images.

Of greater interest is the diffracted solution given by Eqs. (5.13) and (5.14) for $t>\tau_{0}$

$\frac{\partial \varphi}{\partial t}=\frac{\alpha}{\pi \zeta} \frac{1}{r r_{0} \sinh \eta} \sum_{n=0}^{\infty}$

$$
\begin{aligned}
& \times \cos \frac{n \pi}{\zeta} \theta_{0} \cos \frac{n \pi}{\zeta} \theta \sin \frac{n \pi}{\zeta} \pi e^{-n \pi / \zeta \eta} 1\left(t-\frac{R}{\alpha}\right) \\
& =S 1\left(t-\frac{R}{\alpha}\right) \text {, }
\end{aligned}
$$

where

$$
R=\left[\left(r+r_{0}\right)^{2}+z^{2}\right]^{\frac{1}{2}} .
$$

We see that this solution disappears for $\zeta=\pi / m, m$ an integer, in accord with our previous statements. This 
series may be summed. Writing

$$
\begin{gathered}
\cos \frac{n \pi}{\zeta} \theta_{0} \cos \underset{\zeta}{\zeta}-\sin \frac{n \pi}{\zeta} \pi=\frac{1}{8 i}\left(e^{i(n \pi / \zeta) \theta_{0}}+e^{-i(n \pi / \zeta) \theta_{0}}\right) \\
\quad \times\left(e^{i(n \pi / \zeta) \theta}+e^{-i(n \pi / \zeta) \theta}\right)\left(e^{i(n \pi / \zeta) \pi} e^{-i(n \pi / \zeta) \pi}\right)
\end{gathered}
$$

Eq. (5.22) becomes a sum of eight infinite geometric series, each of which can be summed. Collecting conjugate terms in pairs, one has the final result

$$
\begin{aligned}
& \frac{\partial \varphi}{\partial t}=\frac{\alpha}{4 \pi \zeta} \frac{1}{r r_{0} \sinh \eta} e^{-\pi \eta / \zeta} \\
& \quad \times\left[\frac{\sin (\pi / \zeta)\left(\pi \pm \theta \pm \theta_{0}\right)}{1-2 e^{-\pi \eta / \zeta} \cos (\pi / \zeta)\left(\pi \pm \theta \pm \theta_{0}\right)+e^{-2 \pi \eta / \zeta}}\right] 1\left(t-\frac{R}{\alpha}\right),
\end{aligned}
$$

where the \pm signs are again used for conciseness, to show that the form in brackets is actually a sum of four terms corresponding to all possible combinations of these signs. Applying the operation $-\rho \partial / \partial t$ we obtain the diffracted pressure wave

$$
\begin{aligned}
p & =\frac{\rho \alpha^{3}}{4 \pi \zeta} \frac{t}{r^{2} r_{0}^{2} \sinh ^{2} \eta} e^{-\pi \eta / \zeta} \\
& \times\left[\operatorname{cotanh} \frac{\sin (\pi / \zeta)\left(\pi \pm \theta \pm \theta_{0}\right)}{1-2 e^{-\pi \eta / \zeta} \cos (\pi / \zeta)\left(\pi \pm \theta \pm \theta_{0}\right)+e^{-2 \pi \eta / \zeta}}\right. \\
+ & \left.\frac{\pi}{\zeta} \frac{\left(1-e^{-2 \pi \eta / \zeta}\right) \sin (\pi / \zeta)\left(\pi \pm \theta \pm \theta_{0}\right)}{\left[1-2 e^{-\pi \eta / \zeta} \cos (\pi / \zeta)\left(\pi \pm \theta \pm \theta_{0}\right)+e^{-2 \pi \eta / \zeta}\right]^{2}}\right] \\
& \times 1\left(t-\frac{R}{\alpha}\right)-\rho S \delta\left(t-\frac{R}{\alpha}\right) .
\end{aligned}
$$

Note that this solution is explicit, closed and involves elementary functions only. The second term has only been included for completeness. Usually it will play no role, since for other transients the source function will in general be specified in terms of pressure versus time, and one will apply Duhamel's theorem directly to Eqs. (5.22) or (5.24) to obtain the pressure wave.

By virtue of Eqs. (5.14) and (5.6), we see that for $t=\tau_{0}, \eta=0$. Therefore the diffracted pressure front arrives with infinite amplitude at time $t=\tau_{0}$. Its sign depends upon the particular choice of $\theta, \theta_{0}, \zeta$ as may be seen by examining the behavior of the trigonometric terms in the brackets for $t=\tau_{0}$. In other words the diffracted wave may start either as a compression or as a rarefaction, depending upon the particular geometry considered. This is illustrated by the numerical results of Fig. 5 showing the form of the diffracted pressure wave for various wedges and corners for source and receiver situated at $r=r_{0}$ and on the same or opposite walls: the wave diffracted backwards towards the source may be either compressional or rarefactional and is of opposite phase to the wave diffracted along the other wall. In all cases the pressure amplitude is doubly infinite at $t=\tau_{0}$, after which it decreases exponentially. This exponential tail is due in part to the fact that the diffracted arrival is radiated by a line source (the wedge apex) and thus exhibits this typical feature of two-dimensional wave propagation, and in part to the time differences between the contributions of different points of this line. After the direct pressure wave has arrived at the point of the apex $r=0, z=0$ closest to source, we may visualize the process of diffraction as due to the radiation by a disturbance
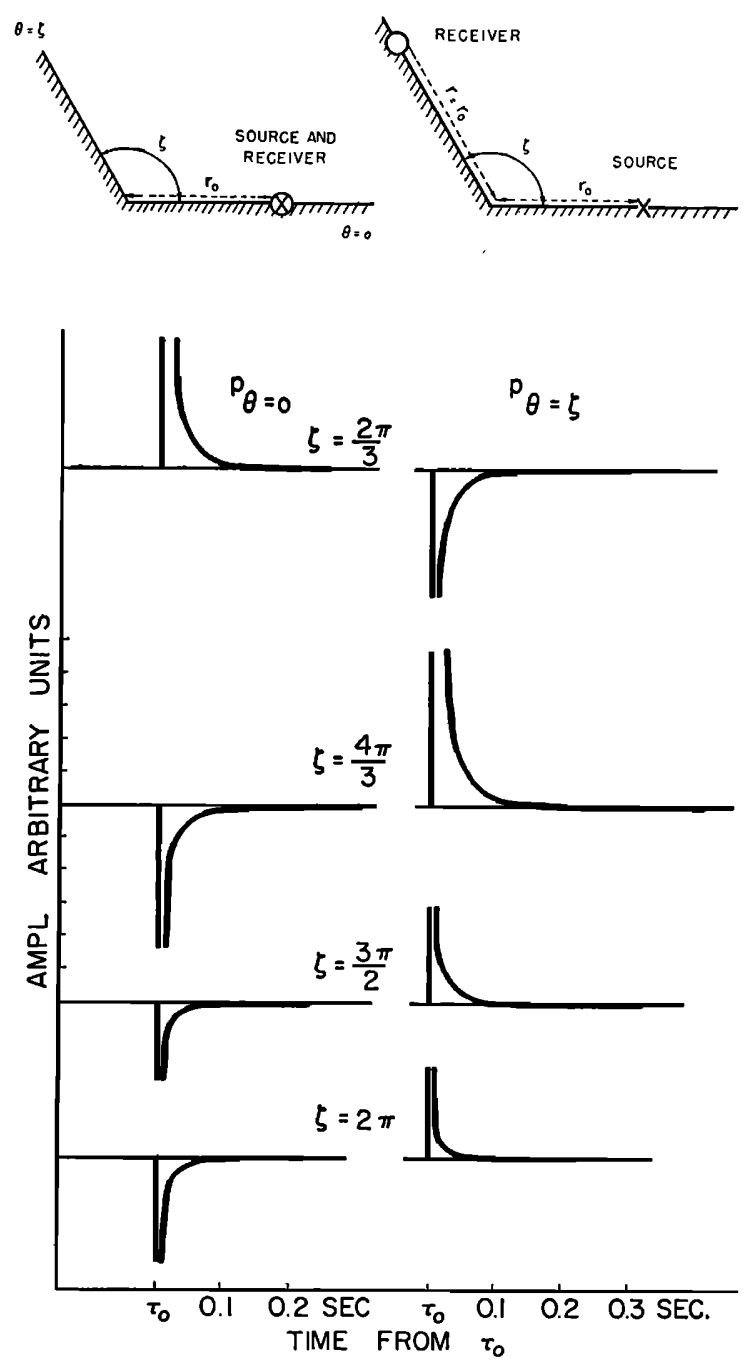

Fig. 5. Comparison of the diffracted wave due to an instantaneous explosive source at $r=r_{0}$, for various values of the corner or wedge angle $\zeta$. On the left side we have shown the shape of the wave when the source and receiver positions coincide. On the right we have shown it for receiver and source on opposite walls, at the same distance $r=r_{0}$ from the apex. $\tau_{0}=0$ is the instant of arrival of the diffracted wave front. Compression is plotted downwards, dilation is upwards. The delta function term of Eq. (5.25) cannot be plotted -it contributes an infinite pressure at $t=\tau_{0}$, of opposite sign to that marking the beginning of the exponential tail shown here. 
traveling with supersonic velocity along the summit line of the wedge.

Equations (5.25) and (5.24) exhibit the interesting feature that for certain values of $\theta, \theta_{0}$, and $\zeta$ no diffracted solution exists even when $\zeta$ is not an integral submultiple of $\pi$, e.g., if $\zeta=2 \pi /(2 \kappa+1), \theta_{0}=\pi /(2 \kappa+1)$. In this case the source is in the bisecting plane, and each half of the wedge or corner is an integral submultiple of $\pi$ : the solution is obtained by juxtaposing two wedges of angle $\zeta=\pi /(2 \kappa+1)$ with the source on the common wall.

These considerations, combined with the results of Fig. 5 show that a more thorough numerical study of this problem is desirable, although it would be out of place here.

Note that the series in Eq. (5.22) converges rapidly for $t \gg \tau_{0}$ and may often be easier to use for numerical purposes than the closed forms (5.24) and (5.25).

The formulas for a wedge with free surfaces, or one free and one rigid surface are equally easy to obtain by replacing the cosine in Eq. (5.1) by a sine or a suitable combination of sines and cosines. Finally, it is clear that this approach should lead directly to new solutions of the diffraction problem for transient point sources in all separable coordinate systems; e.g., diffraction by spheres, cylinders, circular apertures, etc.

\section{CONCLUSIONS}

We have endeavored to establish and to some extent illustrate the Lagrangian technique of using normal coordinates in the context of infinite spaces subject to the laws of mechanics. This method, in Hamiltonian form, is also used in the classical and quantum theories of electrodynamics. To our knowledge, it has never been applied to problems of acoustic and elastic wave propagation. As noted in Sec. 1, this is probably due in part to the great impetus given to the linear operator approach by the advent of quantum mechanics early in this century. It has been our purpose to reestablish the normal coordinate point of view in propagation problems, the development of which since Rayleigh's time had stopped with the theory of enclosures, i.e., vibrations. As we have seen there are no essential difficulties in extending it to infinite media. Indeed, this had already been done in field theory, both in vacuum electrodynamics ${ }^{1}$ and in problems involving Čerenkov radiation..$^{22}$

We have followed a straightforward path, staying at all times within the realm of mechanics. Within this framework our derivations have been rigorous, with the possible exception of Eqs. (2.14), (2.15), and (2.16). However we stressed the fact that these are purely a shorthand for the standard procedure of counting modes which is the accepted procedure in

\footnotetext{
${ }^{22}$ J. G. Linhart, J. Appl. Phys. 26, 527 (1955).
}

field theory, leading to identical results. This latter technique is perhaps also not above reproach from the mathematical standpoint, but no physicist would question its validity.

One should note that, from the formal standpoint, the method of normal coordinates as developed here is somewhat more general than is implied by its applications to field theory. For instance, mechanical continua are often described by higher order operators and complex boundary conditions of a type not encountered in field theory.

The generality of this method is also apparent in the key equations (2.13) (diagonalization or orthonormality condition) and (3.4) or (3.5) (generalized force for arbitrary sources), which are valid even in nonseparable coordinate systems, as is clear from their three-dimensional vector form. In this lies a clear potential advantage of this approach, which has, however, not been exploited in this paper.

For the solution of simple harmonic problems in separable systems this method has nothing to especially recommend it over the usual methods of Green's function and linear operators.

For the solution of transient problems in simple cases, it does not have obvious advantages over the Laplace transform method. But in complicated cases, it is simpler and more direct. That this is so, begins to be apparent in the diffraction problem of Sec. 5. In complex problems of heterogeneous systems of fluid or solid elastic layers it has in principle the advantage of discriminating between various types of modes. For example, in a simple homogeneous elastic half-space one has, roughly speaking, a family of exponential modes corresponding to Rayleigh waves, and two families of sinusoidal modes corresponding to body waves. It is clear, from the general formulas of Secs. 2 and 3 that in this method the effect of a given source upon each family of modes is perforce obtained separately. These effects are then summed to obtain the total result. But, in the Laplace transform method this is not possible - the solutions contain all effects together, and they are not separated from the start.

Finally, somewhat along the same lines it may be worthwhile noting that it is sometimes possible to isolate certain predominant modes which may be intuitively predicted or apparent from test data. Such phenomena exhibit hybrid features of vibration and propagation. The present method furnishes an ideal tool for their treatment.

Most of these points must, of course, await future elaboration by means of specific examples. The object of this paper was chiefly expository and eclectic. We thought it inadvisable to go beyond the relatively simple example of Sec. 5. 